\title{
The association between prevalent vertebral fractures and bone quality of the distal radius and distal tibia as measured with HR-pQCT in postmenopausal women with a recent non-vertebral fracture at the Fracture Liaison Service
}

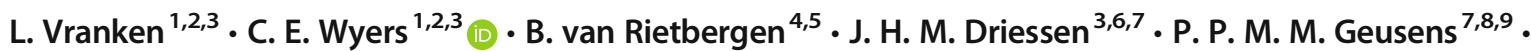 \\ H. M. J. Janzing ${ }^{10} \cdot$ R. Y. van der Velde ${ }^{1,2,3}$. J. P. W. van den Bergh ${ }^{1,2,3,9,10}$
}

Received: 17 October 2018 / Accepted: 3 July 2019 / Published online: 17 July 2019

(C) The Author(s) 2019

\begin{abstract}
Summary We evaluated the association between prevalent vertebral fractures and bone micro-architecture and strength measured using HR-pQCT in postmenopausal women with a recent non-vertebral fracture visiting the Fracture Liaison Service. The presence and severity of prevalent vertebral fracture reflect generalized bone deterioration.

Introduction We evaluated the association between prevalent vertebral fractures (VFs) and bone micro-architecture and strength measured using HR-pQCT in postmenopausal women visiting the Fracture Liaison Service.

Methods In this cross-sectional study in women aged 50-90 with a recent non-vertebral fracture (NVF), VFs were identified on lateral spine images by dual-energy X-ray absorptiometry. Bone micro-architecture and strength were measured at the nondominant distal radius and distal tibia using HR-pQCT. Linear regression analyses were used to estimate the association between prevalent VFs and HR-pQCT parameters.

Results We included 338 women of whom 74 (21.9\%) women had at least one prevalent VF. After adjustment for femoral neck aBMD (FN aBMD) and other parameters, women with at least one prevalent vertebral fracture had significantly lower total and trabecular vBMD and trabecular number $(\beta-16.7,-11.8$, and -7.8 in the radius and $-21.4,-16.6$, and -7.2 in the tibia, respectively), higher trabecular separation at the radius and tibia ( $\beta 9.0$ and 9.3, respectively), and lower cortical thickness and calculated ultimate failure load and compressive bone strength at the tibia $(\beta-5.9,-0.6$, and -10.9 , respectively) as compared with those without prevalent VFs. Furthermore, more severe prevalent VFs were associated with even lower total and trabecular vBMD and lower ultimate failure load and compressive stiffness at the radius and tibia, and lower trabecular number and higher trabecular separation at the radius.

Conclusion This study indicates that the presence and severity of prevalent VFs reflect generalized bone deterioration in women with a recent NVF, independently of FN aBMD.

J. P. W. van den Bergh

jvdbergh@viecuri.nl

1 Department of Internal Medicine, VieCuri Medical Center, P.O. Box 1926, 5900 BX Venlo, The Netherlands

2 Department of Internal Medicine, Maastricht University Medical Center +, P.O. Box 616, 6200 MD Maastricht, The Netherlands

3 NUTRIM School of Nutrition and Translational Research in Metabolism, Maastricht University, Maastricht, The Netherlands

4 Faculty of Biomedical Engineering, Eindhoven University of Technology, Eindhoven, The Netherlands

5 Department of Orthopaedic Surgery, Maastricht University Medical Center +, P.O. Box 616, 6200 MD Maastricht, The Netherlands

6 Department of Clinical Pharmacy and Toxicology, Maastricht University Medical Center +, P.O. Box 616, 6200 MD Maastricht, The Netherlands

7 CAPHRI School for Public Health and Primary Care, Maastricht University, Maastricht, The Netherlands

8 Department of Internal Medicine, Subdivision Rheumatology, Maastricht University Medical Center +, P.O. Box 616, 6200 MD Maastricht, The Netherlands

9 Biomedical Research Center, Hasselt University, Agoralaan, Gebouw D, 3590 Diepenbeek, Belgium

10 Department of Surgery, VieCuri Medical Center, P.O. Box 1926, 5900 BX Venlo, The Netherlands
\end{abstract}


Keywords Fracture Liaison Service $\cdot$ HR-pQCT $\cdot$ Vertebral fractures

\section{Introduction}

Vertebral fractures (VFs) are the most frequently occurring osteoporotic fractures [1-3]. Because only one-third of patients with VFs present with an acute, symptomatic episode [4], VFs are underdiagnosed [5, 6]. The Dutch guideline on osteoporosis and fracture prevention recommends vertebral fracture assessment (VFA) in all patients aged 50 years and older with a recent non-vertebral fracture (NVF) [7]. In patients with a recent NVF at the Fracture Liaison Service (FLS), prevalent VFs have been reported in $20-26 \%$ of patients [8-10] and moderate or severe prevalent VFs in $15-17 \%$ [8, $10]$.

Prevalent VFs have been positively associated with subsequent VFs and NVFs, independent of age and bone mineral density (BMD) [11-15]. Increased fracture risk may be caused by other factors not captured by BMD measurements, such as bone micro-architecture and bone strength. Indeed, previous cross-sectional studies have shown that, compared with subjects without a VF, patients with prevalent VFs have significantly impaired bone micro-architecture of trabecular and cortical bone in the distal radius and tibia after adjustment for BMD in the spine or hip [16-19]. Furthermore, in previous prospective studies, deterioration of HR-pQCT indices of trabecular and cortical bone and lower calculated bone strength improve prediction of fracture beyond femoral neck areal BMD or FRAX scores alone [20-24].

The abovementioned cross-sectional studies compared patients with prevalent VFs to fracture-free controls [16-19]. Additionally, Stein et al. [18] compared women with a prevalent VF with women with a NVF and reported significantly greater deterioration of bone micro-architecture at the tibia in those with VFs. Currently, there are no studies evaluating whether the presence of a prevalent VF is associated with impaired bone micro-architecture in the presence of a recent NVF. We therefore evaluated the association between prevalent VFs and bone micro-architecture and strength in the distal radius and distal tibia measured using HR-pQCT in postmenopausal women visiting the FLS after a recent NVF.

\section{Materials and methods}

\section{Subject and study procedures}

Data from the FX MoVie study, an ongoing prospective observational study, were used. The primary objective of this study is to assess bone structure parameters and bone strength by HR-pQCT and physical activity in relation to falls, fractures, and mortality in patients with a recent clinical fracture. Included were 500 patients aged between 50 and 90 years with a recent, radiologically confirmed clinical vertebral or non-vertebral fracture, who visited the FLS of VieCuri Medical Center in The Netherlands, and who were willing and able to participate. Excluded were non-Caucasian patients; patients with a fracture due to high-energy trauma, bone metastasis, failure of prosthesis, or osteomyelitis; and patients with cognitive impairment.

The study protocol (registration number NL45707.072.13) was approved by an independent Medical Ethics Committee and complied with the Declaration of Helsinki. All patients gave written informed consent prior to participation.

The present cross-sectional study includes baseline data of postmenopausal women with a recent NVF. Patients who presented with a symptomatic VF were excluded. The mean time between NVF and baseline assessment was $4.2 \pm 1.1$ months. Baseline assessment included a detailed questionnaire for evaluation of risk factors for osteoporosis, falls, and fractures; laboratory tests to detect contributors to secondary osteoporosis and metabolic bone disease; BMD measurement and lateral imaging of the spine by dual-energy X-ray absorptiometry (DXA); and HR-pQCT scans of the distal radius and tibia. Fractures were categorized according to FRAX into major osteoporotic fractures (except clinical vertebral fractures which were excluded from this study) or all other fractures [25].

\section{Areal bone mineral density}

Areal bone mineral density (aBMD) was measured at the hip and lumbar spine by DXA using the Hologic QDR 4500 (Hologic, Bedford, MA, USA). Lumbar spine evaluation was performed according to the International Society of Clinical Densitometry (ISCD) criteria (https://www.iscd.org/ official-positions/6th-iscd-position-development-conferenceadult). Vertebrae with grade 2 or 3 deformities according to Genant [26] were excluded and lumbar spine aBMD was determined based on the remaining vertebrae. Lumbar spine evaluation was based on at least two vertebrae.

Osteoporosis was diagnosed according to the World Health Organization (WHO) criteria for BMD [27]. Patients were classified according to the lowest value of T-score in femoral neck, total hip, or lumbar spine. T-scores of $\leq-2.5$ standard deviations (SD) below the reference mean were classified as osteoporosis, T-scores between -1.0 and $-2.5 \mathrm{SD}$ were classified as osteopenia, and T-scores $\geq-1.0 \mathrm{SD}$ were classified as normal. 


\section{Vertebral fracture assessment}

Prevalent vertebral fractures were identified on lateral spine images made with DXA. According to the semi-quantitative method of Genant [26], VFs were graded as mild (grade 1, height loss between 20 and 25\%), moderate (grade 2, height loss between 25 and $40 \%$ ), or severe (grade 3 , height loss > $40 \%$ ). Patients were classified according to the most severe $\mathrm{VF}$ as those without VFs, those with at least one mild VF, or those with at least one moderate or severe VF. Vertebral deformities related to other conditions, such as Scheuermann's disease, degenerative disease, and Schmorl's nodes, were not classified as prevalent VF.

\section{HR-pQCT imaging}

The non-dominant radius was scanned using a secondgeneration HR-pQCT scanner (XtremeCT II; Scanco Medical AG, Brüttisellen, Switzerland) using the standard in vivo protocol as provided by the manufacturer (effective energy of $68 \mathrm{kVp}$, tube current of $1470 \mu \mathrm{A}$, and integration time of $43 \mathrm{~ms}$ ) unless the patient had previously sustained a distal radius fracture at the non-dominant site; then, the dominant site was scanned. The distal tibia was scanned at the same site as the distal radius unless that site was previously fractured; then, the other site was scanned. The forearm and lower leg were placed into a carbon fiber cast. The region of interest was determined based on an anteroposterior scout projection of the scan site. A reference line was placed on the distal radius and distal tibia joint surface. The scan started $9.0 \mathrm{~mm}$ from the reference line in the proximal direction and spanned $10.2 \mathrm{~mm}$ in length. Images were reconstructed using an isotropic voxel size of $61 \mu \mathrm{m}$, resulting in 168 consecutive slices. Each scan was graded for motion-induced image artifacts by the operator according to the manufacturer's guideline and as described by Pialat et al. [28]. In case the images were of insufficient quality, i.e., grade 4 or 5 , the scan was repeated with a maximum up to two times. Only scans with quality 1 to 3 were used for analyses in this study.

\section{Image analysis of HR-pQCT scans}

All scans were evaluated using the standard patient evaluation protocol as provided by the manufacturer. We used a fully automated segmentation method, which uses two thresholds and a series of morphological dilatation and erosions to extract the endosteal and periosteal surface of the cortex [29]. This is based on the assumption that the trabecular region is enclosed by the cortical region. The periosteal contour was automatically derived and manually modified by a single operator when contours visually deviated from the periosteal boundary. The following bone parameters were measured: volumetric bone mineral density $\left(\mathrm{mgHA} / \mathrm{cm}^{3}\right)$ was assessed for the total region (Dtot) and trabecular region (Dtrab) and cortical region (Dcort) separately. For the trabecular region, the microarchitectural parameters trabecular bone volume fraction (Tb.BVTV) (\%), trabecular number (Tb.N) $\left(\mathrm{mm}^{-1}\right)$, trabecular thickness (Tb.Th) (mm), and trabecular separation (Tb.Sp) $(\mathrm{mm})$ were measured. For the cortical region, cortical perimeter (Ct.Pm) (mm), cortical thickness (Ct.Th) (mm), cortical porosity (Ct.Po) (\%), and cortical pore diameter (Ct.Po.Dm) $(\mathrm{mm})$ were measured.

Micro-finite element models were generated directly from the segmented HR-pQCT images $[30,31]$ by converting voxels representing bone tissue into brick elements of the same size. Young's modulus of $10 \mathrm{GPa}$ and Poisson's ratio of 0.3 were assigned to every element. Compression stiffness and estimated failure load were determined by simulating a "high-friction" compression test in the axial direction [30].

\section{Statistical analysis}

General characteristics and mean HR-pQCT parameters were compared between women with and without prevalent VFs using the independent Student's $t$ test for continuous variables and the chi-square test or Fisher's exact test for categorical variables. Further, mean HR-pQCT parameters were compared between women according to prevalent VF severity (none vs. mild (grade 1) vs. moderate or severe (grades 2-3)) using one-way ANOVA with the Bonferroni correction. Descriptives are provided as mean \pm SD for continuous variables and number $(\%)$ for categorical variables. Log transformation was performed if variables showed a skewed distribution. Multiple linear regression analysis was used to estimate the association between VF and HR-pQCT parameters, yielding unstandardized beta $(\beta)$ and $95 \%$ confidence interval (CI). Potential confounders were included in the analyses if they independently changed the beta coefficient for VF by at least $5 \%$. All regression analyses were adjusted for age, height, weight, type of recent NVF (major osteoporotic fracture, i.e., hip, proximal humerus, and distal radius fractures), previous fractures at or above the age of 50 years, self-reported use of anti-osteoporosis treatment (never vs. history vs. current), and femoral neck aBMD. A $P$ value $\leq .05$ was considered statistically significant for general characteristics, and a $P$ value $\leq .002$ for HRpQCT parameters. Analyses were conducted using SPSS for Mac (version 24.0, IBM SPSS Statistics, USA).

\section{Results}

In total, 338 postmenopausal women with a NVF were included in this study (Fig. 1). HR-pQCT scans were not performed in 11 women at the radius, and in 5 women at 
Fig. 1 Flowchart of patient inclusion

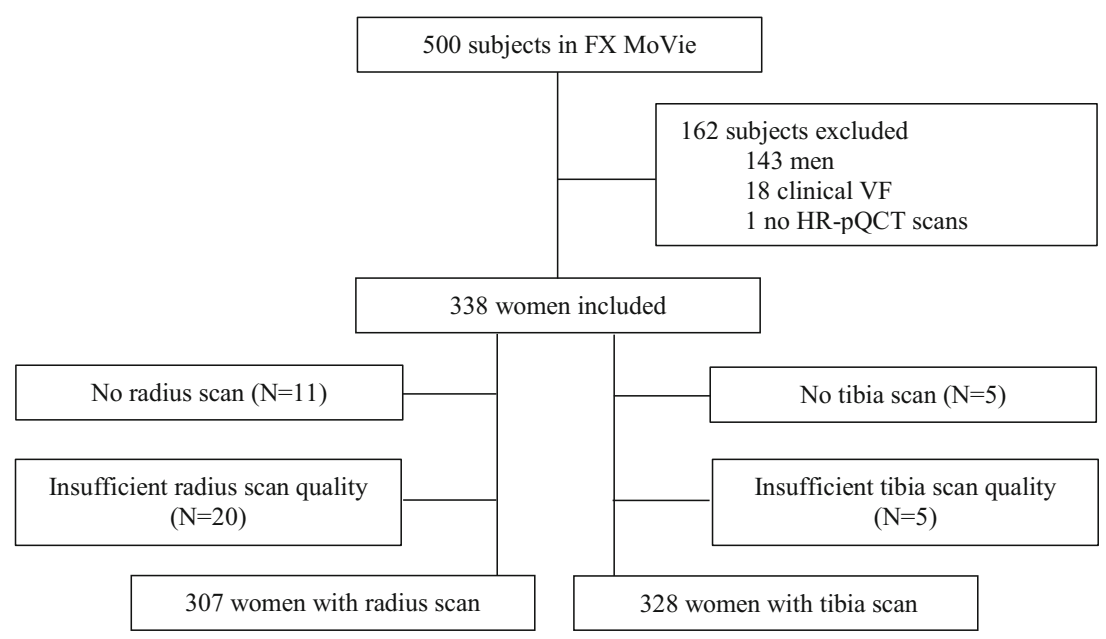

the tibia because of bilateral fractures. Additionally, 20 radius and 5 tibia scans were excluded because of insufficient scan quality due to motion artifacts, resulting in 307 radius and 328 tibia scans that were included in the analyses.

Of the 338 women, 74 (21.9\%) had at least one prevalent VF and 264 had no prevalent VF. General characteristics of women according to their prevalent VF status are shown in Table 1. Compared with women without a prevalent VF, women with at least one prevalent VF were older, had lower femoral neck (FN) and total hip (TH) aBMD, were more likely to have had a previous fracture at or above the age of 50 years, and were more likely to have ever used anti-osteoporosis treatment.

\section{HR-pQCT parameters according to prevalent VF status}

The mean unadjusted HR-pQCT parameters at the radius and tibia for women according to presence and severity of prevalent VFs are shown in Table 2. Compared with women without a prevalent VF, those with at least one prevalent VF had lower total and trabecular vBMD, trabecular number, ultimate failure load, and compression stiffness, and higher trabecular separation at both the radius and tibia, and lower cortical thickness at the tibia.

Results of linear regression analyses examining the association between prevalent VF status and HR-pQCT parameters at the radius and tibia are shown in Table 3 . In the adjusted analyses, at least one prevalent VF was associated with lower total and trabecular vBMD and trabecular number $(\beta-1.6,-11.8$, and -7.8 in the radius and $-21.4,-$ 16.6 , and -7.2 in the tibia, respectively), and higher trabecular separation at the radius and tibia ( $\beta 9.0$ and 9.3, respectively), and lower cortical thickness, ultimate failure load, and compression stiffness at the tibia $(\beta-5.9,-0.6$ and -10.9 , respectively).

\section{HR-pQCT parameters according to prevalent VF severity}

Compared with women without prevalent VFs, those with at least one moderate or severe prevalent VF had lower total and trabecular vBMD, trabecular number, ultimate failure load, and compression stiffness, and higher trabecular separation at both the radius and tibia (Table 2). Further, a similar pattern was found when patients with at least one mild prevalent VF were compared with those without prevalent VF, with the exception that ultimate failure load and compression stiffness at the radius, and trabecular number and separation at the tibia were not significantly different for these two groups (Table 2).

Adjusted regression analyses showed that total and trabecular vBMD, ultimate failure load, and compression stiffness were lower at the radius and tibia $(\beta-26.10,-17.6,-0.3$, and -4.6 in the radius and $-27.6,-20.0,-0.7$, and -13.2 in the tibia, respectively) and trabecular number was lower $(\beta-$ $10.9)$ and trabecular separation was higher $(\beta 14.4)$ at the radius in women with moderate or severe prevalent VF as compared with those without prevalent VF (Table 3). Further, in women with at least one mild prevalent VF, total and trabecular vBMD and calculated ultimate failure load and compressive bone strength were lower at the tibia $(\beta-16.4,-13.1,-0.5$, and -9.1 , respectively) than in those without prevalent VF.

In addition, there was a significant trend analysis for lower total and trabecular vBMD, trabecular number, ultimate failure load, and compression stiffness, and higher trabecular separation at the radius and tibia, and lower cortical thickness at the tibia with increasing prevalent VF severity (Table 3).

\section{Discussion}

In this study, in postmenopausal women attending the FLS with a recent NVF, the presence of at least one prevalent VFs was independently associated with lower total and 
Table 1 General characteristics of patients with a recent NVF at the FLS according to the presence of prevalent vertebral fracture

\begin{tabular}{|c|c|c|c|}
\hline & \multicolumn{3}{|l|}{ Women $(n=338)$} \\
\hline & No VF $(n=264)$ & $\geq 1$ VF Gr. $\geq 1(n=74)$ & $P$ value \\
\hline Age (years) & $62.9 \pm 7.8$ & $67.7 \pm 8.4$ & $.000 *$ \\
\hline Height $(\mathrm{cm})$ & $1.6 \pm 0.1$ & $1.6 \pm 0.1$ & .295 \\
\hline Weight $(\mathrm{kg})$ & $74.6 \pm 14.3$ & $72.7 \pm 12.1$ & .296 \\
\hline BMI $\left(\mathrm{kg} / \mathrm{m}^{2}\right)$ & $27.6 \pm 5.0$ & $27.1 \pm 4.0$ & .474 \\
\hline FRAX major osteoporotic fractures & $82(31.1)$ & $30(40.5)$ & .126 \\
\hline LS aBMD $\left(\mathrm{g} / \mathrm{cm}^{2}\right)$ & $0.93 \pm 0.14$ & $0.91 \pm 0.17$ & .388 \\
\hline $\mathrm{FN}$ aBMD $\left(\mathrm{g} / \mathrm{cm}^{2}\right)$ & $0.70 \pm 0.11$ & $0.67 \pm 0.10$ & $.032 *$ \\
\hline TH aBMD $\left(\mathrm{g} / \mathrm{cm}^{2}\right)$ & $0.85 \pm 0.13$ & $0.81 \pm 0.12$ & $.028 *$ \\
\hline $\begin{array}{l}\text { Normal BMD } \\
\text { Osteopenia }\end{array}$ & $\begin{array}{l}72(27.3) \\
135(51.1)\end{array}$ & $\begin{array}{l}13(17.6) \\
38(51.4)\end{array}$ & .113 \\
\hline Osteoporosis & $57(21.6)$ & $23(31.1)$ & \\
\hline Previous fracture after age 50 years & $48(18.2)$ & $36(48.6)$ & $.000 *$ \\
\hline Parent fractured hip & $17(6.4)$ & $4(5.4)$ & .999 \\
\hline Current smoking & $36(13.6)$ & $9(12.2)$ & .741 \\
\hline Glucocorticoids & $12(4.5)$ & $3(4.1)$ & .999 \\
\hline Rheumatoid arthritis & $10(3.8)$ & $4(5.4)$ & .516 \\
\hline Secondary osteoporosis & $70(26.5)$ & $21(28.4)$ & .749 \\
\hline Alcohol $\geq 3$ units/day & $4(1.5)$ & $1(1.4)$ & .999 \\
\hline History or current use of anti-osteoporosis drugs & $21(8.0)$ & $19(26.0)$ & $.000 *$ \\
\hline Self-reported use of anti-osteoporosis medication, $n(\%)$ & $18(6.9)$ & $18(24.7)$ & $.000 *$ \\
\hline Type, $n(\%)^{\#}$ & & & \\
\hline Alendronic acid & $11(61.1)$ & $9(50.0)$ & .887 \\
\hline Risedronic acid & $5(27.8)$ & $6(33.3)$ & \\
\hline Zoledronic acid & $0(0.0)$ & $1(5.6)$ & \\
\hline Unknown & $2(11.1)$ & $2(11.1)$ & \\
\hline Duration (year), mean $\pm \mathrm{SD}^{\#}$ & $4.5 \pm 2.7$ & $5.2 \pm 4.1$ & .577 \\
\hline Current, $n(\%)^{\#}$ & $3(16.7)$ & $10(55.6)$ & $.015^{*}$ \\
\hline History, $n(\%)^{\#}$ & $15(83.3)$ & $8(44.4)$ & \\
\hline Time since cessation of treatment (year), mean $\pm \mathrm{SD}^{\#}$ & $2.6 \pm 2.6$ & $1.1 \pm 2.3$ & .109 \\
\hline Falls past year & $79(30.0)$ & $21(28.8)$ & .834 \\
\hline
\end{tabular}

Variables are presented as mean \pm standard deviation or number (percentage). $P$ value $<0.05$ is considered significant $(*)$

" In patients who have ever used anti-osteoporosis medication

$V F$, vertebral fracture; $B M I$, body mass index; $L S$, lumbar spine; $F N$, femoral neck, $T H$, total hip; $a B M D$, areal bone mineral density

trabecular vBMD, lower trabecular number, and higher trabecular separation at the radius and tibia and with lower cortical thickness, ultimate failure load, and compressive stiffness at the tibia. Furthermore, moderate or severe prevalent VFs were associated with even lower total and trabecular vBMD and lower ultimate failure load and compressive stiffness at the radius and tibia, and lower trabecular number and higher trabecular separation at the radius.

To the best of our knowledge, there are no studies evaluating the association between prevalent VFs and bone microarchitecture and bone strength in patients with a recent NVF. Previous cross-sectional studies have shown that, compared with fracture-free controls, patients with VFs have significantly impaired bone micro-architecture of trabecular and cortical bone in the distal radius and tibia after adjustment for BMD in the spine or hip [16-19]. Furthermore, Stein et al. [18] reported a significantly greater deterioration of bone micro-architecture in the tibia, but not the radius in women with a VF compared with those with a NVF. However, in the study by Stein et al. [18], only 12 out of 30 women with a VF had a history of a NVF, whereas in our study, all patients had a recent NVF.

In line with our findings, two previous studies reported more bone micro-architectural deterioration with increasing severity of prevalent VFs in women [16, 17], whereas one study found no association between severity of prevalent VFs and HR-pQCT parameters [18]. In contrast to our study, 
Table 2 HR-pQCT parameters at the distal tibia and radius according to presence and severity of prevalent VFs in women with a recent NVF at the FLS

\begin{tabular}{|c|c|c|c|c|c|}
\hline \multirow{3}{*}{$\begin{array}{l}\text { Radius }(n=307) \\
\text { vBMD }\end{array}$} & \multirow[b]{2}{*}{ No VF $(n=240)$} & \multirow{2}{*}{$\begin{array}{l}\text { Presence of prevalent VFs } \\
\geq 1 \text { VF Gr. } \geq 1(n=67)\end{array}$} & \multicolumn{3}{|c|}{ Severity of prevalent VFs } \\
\hline & & & $\geq 1$ VF Gr. $1(n=35)$ & $\geq 1$ VF Gr. $2-3(n=32)$ & $P$ value for trend \\
\hline & \\
\hline Dtot $\left(\mathrm{mgHA} / \mathrm{cm}^{3}\right)$ & $270 \pm 62$ & $233 \pm 55^{*}$ & $236 \pm 56^{*}$ & $230 \pm 55^{*}$ & .000 \\
\hline Dtrab (mgHA/ $\left.\mathrm{cm}^{3}\right)$ & $121 \pm 39$ & $96 \pm 37 *$ & $101 \pm 33^{*}$ & $91 \pm 41 *$ & .000 \\
\hline Dcort (mgHA/cm $\left.{ }^{3}\right)$ & $892 \pm 62$ & $872 \pm 69$ & $870 \pm 64$ & $875 \pm 75$ & .068 \\
\hline \multicolumn{6}{|l|}{ Micro-architecture } \\
\hline Tb.N $\left(\mathrm{mm}^{-1}\right)$ & $1.20 \pm 0.26$ & $1.02 \pm 0.31 *$ & $1.06 \pm 0.28 *$ & $0.97 \pm 0.34 *$ & .000 \\
\hline Tb.Th (mm) & $0.22 \pm 0.02$ & $0.23 \pm 0.02$ & $0.23 \pm 0.02$ & $0.23 \pm 0.02$ & .362 \\
\hline $\mathrm{Tb} . \mathrm{Sp}(\mathrm{mm})$ & $0.86 \pm 0.27$ & $1.15 \pm 0.68 *$ & $1.04 \pm 0.46^{*}$ & $1.28 \pm 0.86^{*}$ & .000 \\
\hline Ct.Po $(\%)$ & $0.86 \pm 0.56$ & $0.88 \pm 0.49$ & $0.86 \pm 0.43$ & $0.90 \pm 0.56$ & .899 \\
\hline Ct.Th (mm) & $0.92 \pm 0.18$ & $0.85 \pm 0.15$ & $0.85 \pm 0.15$ & $0.84 \pm 0.15$ & .012 \\
\hline Ct.Po.Dm (mm) & $0.19 \pm 0.03$ & $0.20 \pm 0.03$ & $0.20 \pm 0.04$ & $0.20 \pm 0.03$ & .359 \\
\hline \multicolumn{6}{|l|}{ Biomechanical } \\
\hline F.Ult $(\mathrm{kN})$ & $2.81 \pm 0.72$ & $2.49 \pm 0.55^{*}$ & $2.58 \pm 0.51$ & $2.40 \pm 0.59 *$ & .002 \\
\hline Scomp $(\mathrm{kN} / \mathrm{mm})$ & $52 \pm 13$ & $47 \pm 10^{*}$ & $49 \pm 9$ & $45 \pm 10^{*}$ & .002 \\
\hline Tibia $(n=328)$ & No VF $(n=256)$ & $\geq 1$ VF Gr. $\geq 1(n=72)$ & $\geq 1$ VF Gr. $1(n=37)$ & $\geq 1$ VF Gr. $2-3(n=35)$ & $P$ value for trend \\
\hline \multicolumn{6}{|l|}{ vBMD } \\
\hline Dtot $\left(\mathrm{mgHA} / \mathrm{cm}^{3}\right)$ & $243 \pm 54$ & $209 \pm 46^{*}$ & $211 \pm 42 *$ & $207 \pm 52 *$ & .000 \\
\hline Dtrab $\left(\mathrm{mgHA} / \mathrm{cm}^{3}\right)$ & $140 \pm 38$ & $119 \pm 34^{*}$ & $121 \pm 31 *$ & $117 \pm 37^{*}$ & .000 \\
\hline Dcort (mgHA/ $\left./ \mathrm{cm}^{3}\right)$ & $837 \pm 73$ & $813 \pm 72$ & $820 \pm 66$ & $806 \pm 77$ & .035 \\
\hline \multicolumn{6}{|l|}{ Micro-architecture } \\
\hline Tb.N $\left(\mathrm{mm}^{-1}\right)$ & $1.19 \pm 0.23$ & $1.08 \pm 0.29^{*}$ & $1.10 \pm 0.28$ & $1.06 \pm 0.30^{*}$ & .004 \\
\hline Tb.Th (mm) & $0.25 \pm 0.02$ & $0.25 \pm 0.02$ & $0.25 \pm 0.02$ & $0.25 \pm 0.02$ & .405 \\
\hline $\mathrm{Tb} . \mathrm{Sp}(\mathrm{mm})$ & $0.87 \pm 0.33$ & $1.02 \pm 0.45^{*}$ & $0.99 \pm 0.44$ & $1.05 \pm 0.46^{*}$ & .004 \\
\hline Ct.Po $(\%)$ & $3.22 \pm 1.32$ & $3.22 \pm 1.37$ & $3.04 \pm 1.12$ & $3.41 \pm 1.59$ & .505 \\
\hline Ct.Th (mm) & $1.21 \pm 0.25$ & $1.10 \pm 0.21 *$ & $1.10 \pm 0.19^{*}$ & $1.11 \pm 0.22$ & .005 \\
\hline Ct.Po.Dm (mm) & $0.23 \pm 0.03$ & $0.23 \pm 0.04$ & $0.23 \pm 0.03$ & $0.24 \pm 0.04$ & .426 \\
\hline \multicolumn{6}{|l|}{ Biomechanical } \\
\hline F.Ult $(\mathrm{kN})$ & $8.00 \pm 1.62$ & $7.14 \pm 1.49^{*}$ & $7.27 \pm 1.35^{*}$ & $7.00 \pm 1.63 *$ & .000 \\
\hline Scomp (kN/mm) & $147 \pm 32$ & $131 \pm 28 *$ & $133 \pm 26^{*}$ & $128 \pm 31 *$ & .000 \\
\hline
\end{tabular}

Variables are presented as mean \pm SD. $P$ value $<0.05$ is considered significant

$* P<0.05$ compared with patients without a prevalent VF (with Bonferroni correction for multiple testing)

$V F$, vertebral fracture; $v B M D$, volumetric bone mineral density; Dtot, total density; Dtrab, trabecular density; Dcort, cortical density; Tb.N, trabecular number; Tb.Th, trabecular thickness; Tb.Sp, trabecular separation; Ct.Th, cortical thickness; Ct.Po, cortical porosity; Ct.Po.Dm, cortical pore diameter; F.Ult, ultimate failure load; Scomp, compression stiffness

all three studies compared women with prevalent VFs with fracture-free controls.

The short time since the NVF is important, because patients with a fracture have an increased risk of subsequent fractures, which is highest immediately after the fracture. This imminent subsequent fracture risk emphasizes the need for immediate and accurate secondary fracture prevention. The fact that the presence of a vertebral fracture in postmenopausal women with a NVF at the FLS is associated with impaired bone quality compared with not having a VF may indicate that patients with a prevalent VF are even at higher subsequent fracture risk.
Interestingly, we found no difference in LS aBMD between women with at least one prevalent VF in addition to a NVF and those without VF, whereas previous studies reported lower LS aBMD in (asymptomatic and/or symptomatic) VF patients than in fracture-free controls. Similarly to our results, Stein et al. [18] reported that there was no difference in LS aBMD when vertebral subjects were compared with NVF subjects. One explanation could be that in NVF patients, the presence of prevalent VFs is not associated with lower LS aBMD. Another explanation could be the impact of degenerative changes in the lumber 
Table 3 Associations between the presence, severity, and number of prevalent vertebral fractures and HR-pQCT parameters in women with a recent NVF at the FLS

\begin{tabular}{|c|c|c|c|c|c|}
\hline & & Presence of prevalent VFs & Severity of prevalent VFs & & \\
\hline Radius & No VF & $\mathrm{VF}(\beta(95 \% \mathrm{CI}))$ & Gr. 1 VF $(\beta(95 \% \mathrm{CI}))$ & Gr. $2-3$ VF $(\beta(95 \% \mathrm{CI}))$ & $P$ value for trend \\
\hline \multicolumn{6}{|l|}{ vBMD } \\
\hline Dtot $\left(\mathrm{mgHA} / \mathrm{cm}^{3}\right)$ & Ref. & $-16.66(-31.93,-1.39)^{*}$ & $-10.05(-28.65,8.56)$ & $-26.10(-47.65,-4.56)^{*}$ & $.015^{*}$ \\
\hline Dtrab $\left(\mathrm{mgHA} / \mathrm{cm}^{3}\right)$ & Ref. & $-11.75(-21.91,-1.58)^{*}$ & $-7.66(-20.06,4.73)$ & $-17.57(-31.92,-3.22)^{*}$ & $.012^{*}$ \\
\hline Dcort $\left(\mathrm{mgHA} / \mathrm{cm}^{3}\right)$ & Ref. & $-9.88(-26.38,6.62)$ & $-7.58(-27.74,12.58)$ & $-13.16(-36.50,10.18)$ & .216 \\
\hline \multicolumn{6}{|l|}{ Micro-architecture } \\
\hline Tb.N (1/mm) & Ref. & $-7.83(-15.19,-0.46)^{*}$ & $-5.50(-14.49,3.49)$ & $-11.15(-21.56,-0.75)^{*}$ & $.024 *$ \\
\hline Tb.Th (mm) & Ref. & $0.20(-0.27,0.67)$ & $0.16(-0.41,0.74)$ & $0.25(-0.42,0.91)$ & .400 \\
\hline Tb.Sp (mm) & Ref. & $8.98(0.68,17.29)^{*}$ & $5.21(-4.91,15.33)$ & $14.37(2.66,26.08)^{*}$ & $.014^{*}$ \\
\hline Ct.Po $(\%)$ & Ref. & $3.26(-16.09,22.61)$ & $0.07(-23.57,23.70)$ & $7.82(-19.55,35.19)$ & .628 \\
\hline Ct.Th (mm) & Ref. & $-3.10(-7.70,1.51)$ & $-1.62(-7.24,4.00)$ & $-5.21(-11.71,1.30)$ & .117 \\
\hline Ct.Po.Dm (mm) & Ref. & $3.41(-1.59,8.41)$ & $2.24(-3.86,8.34)$ & $5.08(-1.99,12.14)$ & .136 \\
\hline \multicolumn{6}{|l|}{ Biomechanical } \\
\hline F.Ult $(\mathrm{kN})$ & Ref. & $-0.15(-0.32,0.02)$ & $-0.08(-0.28,0.13)$ & $-0.26(-0.49,-0.02)^{*}$ & $.038 *$ \\
\hline Scomp $(\mathrm{kN} / \mathrm{mm})$ & Ref. & $-2.47(-5.49,0.55)$ & $-1.02(-4.69,2.66)$ & $-4.55(-8.80,-0.29)^{*}$ & $.045^{*}$ \\
\hline Tibia & No VF & $\mathrm{VF}(\beta(95 \% \mathrm{CI}))$ & Gr. 1 VF $(\beta(95 \% \mathrm{CI}))$ & Gr. $2-3 \mathrm{VF}(\beta(95 \% \mathrm{CI}))$ & $P$ value for trend \\
\hline \multicolumn{6}{|l|}{ vBMD } \\
\hline $\operatorname{Dtot}\left(\mathrm{mgHA} / \mathrm{cm}^{3}\right)$ & Ref. & $-21.35(-33.04,-9.66)^{*}$ & $-16.83(-31.23,-2.42)^{*}$ & $-27.58(-44.02,-11.13)^{*}$ & $.000 *$ \\
\hline Dtrab $\left(\mathrm{mgHA} / \mathrm{cm}^{3}\right)$ & Ref. & $-16.55(-25.22,-7.66)^{*}$ & $-13.88(-24.70,-3.05)^{*}$ & $-19.96(-32.32,-7.61)^{*}$ & $.000 *$ \\
\hline Dcort (mgHA/ $\left./ \mathrm{cm}^{3}\right)$ & Ref. & $-1.65(-19.28,15.97)$ & $3.7(-18.26,25.20)$ & $-8.69(-33.50,16.12)$ & .637 \\
\hline \multicolumn{6}{|l|}{ Micro-architecture } \\
\hline Tb.N (1/mm) & Ref. & $-7.18(-13.69,-0.67)^{*}$ & $-6.81(-14.85,1.22)$ & $-7.68(-16.85,1.49)$ & $.040 *$ \\
\hline Tb.Th (mm) & Ref. & $-0.28(-0.84,0.27)$ & $-0.40(-1.09,0.28)$ & $-0.12(-0.90,0.66)$ & .479 \\
\hline $\mathrm{Tb} . \mathrm{Sp}(\mathrm{mm})$ & Ref. & $9.31(1.92,16.70)^{*}$ & $8.74(-0.38,17.86)$ & $10.09(-0.32,20.50)$ & $.018^{*}$ \\
\hline Ct.Po $(\%)$ & Ref. & $-8.90(-21.20,3.39)$ & $-8.10(-23.28,7.07)$ & $-10.00(-27.32,7.33)$ & .167 \\
\hline Ct.Th (mm) & Ref. & $-5.90(-11.69,-0.11)^{*}$ & $-4.49(-11.63,2.65)$ & $-7.83(-15.99,0.32)$ & $.036^{*}$ \\
\hline Ct.Po.Dm (mm) & Ref. & $0.95(-2.71,4.62)$ & $0.20(-4.32,4.72)$ & $1.99(-3.17,7.15)$ & .492 \\
\hline \multicolumn{6}{|l|}{ Biomechanical } \\
\hline F.Ult (kN) & Ref. & $-0.55(-0.90,-0.21)^{*}$ & $-0.47(-0.89,-0.04)^{*}$ & $-0.67(-1.15,-0.18)^{*}$ & $.002 *$ \\
\hline Scomp (kN/mm) & Ref. & $-10.88(-17.56,-4.21)^{*}$ & $-9.20(-17.43,-0.97)^{*}$ & $-13.20(-22.59,-3.80)^{*}$ & $.001 *$ \\
\hline
\end{tabular}

All analyses are adjusted for age, weight, height, type of recent non-vertebral fracture type (major osteoporotic fracture vs. other fractures), previous fractures at or above the age of 50 years, anti-osteoporosis medication (never vs. history vs. current), and femoral neck areal BMD

No prevalent VF is used as the reference group. *Significant

$V F$, vertebral fracture; Ref., reference; $v B M D$, volumetric bone mineral density; Dtot, total density; Dtrab, trabecular density; Dcort, cortical density; Tb.N, trabecular number; Tb.Th, trabecular thickness; Tb.Sp, trabecular separation; Ct.Th, cortical thickness; Ct.Po, cortical porosity; Ct.Po.Dm, cortical pore diameter; F.Ult, ultimate failure load; Scomp, compression stiffness

spine on the LS aBMD. This should be confirmed and evaluated in future research.

This study has important implications. In women with a recent NVF, the presence of morphometric VF can be used as a marker for generalized bone micro-architecture deterioration, independent of areal BMD and prior fracture. Our results extend previous observations towards women with a NVF, in whom the presence of at least one prevalent VF was associated with impaired trabecular micro-architecture in the radius and tibia, and cortical micro-architecture and bone strength in the tibia.
This study has several limitations. First, this is a crosssectional study. Hence, the interpretation of our findings in the context of subsequent fracture risk in FLS patients cannot be addressed. In previous prospective studies, deterioration of HR-pQCT indices of trabecular and cortical bone and lower bone strength improved prediction of fracture beyond femoral neck areal BMD or FRAX scores alone [20-24]. Future studies are needed to determine the relevance of prevalent $\mathrm{VF}$ in addition to a NVF, in terms of subsequent VF and NVF risk. Second, prevalent VFs were identified on lateral spine images 
by DXA instead of X-ray. The reproducibility of vertebral fracture assessment by DXA is limited, especially for mild (i.e., grade 1) VFs. The inter-rater reliability of absorptiometry was better when only grade 2 or 3 deformities were considered fractured (kappa (95\% CI) $0.640(0.621-0.659)$ ), as compared with when grade 1 deformities were also considered fractures (kappa (95\% CI) 0.560 (0.54100.580)) [32]. Additionally, sensitivity was reported to be $62.5 \%$ and specificity was $93.1 \%$ for grade $2-3$ vertebral deformities, and $51.8 \%$ and $88.7 \%$, respectively, when grade 1 vertebral deformities were also considered fractured [32]. This implies that patients could have been incorrectly classified as mild (i.e., grade 1) VF cases [33]. However, a false-positive VF classification would probably only reduce the HR-pQCT differences between patients with at least one (mild) VF and patients without VFs. Fourth, the reference line for the HR-pQCT scans was placed at a fixed reference point, which resulted in scanning the same region in all patients. However, bone morphology at that region differs between individual patients, where a higher amount of cortical bone will be present in patients with relatively short extremities $[34,35]$. A recent study suggests scanning at a percentage distance of the total length of the bone [36]. Since this information was not available, as an alternative, we have adjusted all the analyses for height. Finally, our results cannot be generalized to the total fracture population, since probably only the most fit and mobile patients were willing and able to visit the FLS and participate in our study, with a healthy complier bias as a consequence.

In conclusion, in this cross-sectional study in postmenopausal women with a recent NVF, the presence and severity of prevalent VF were associated with impaired bone microarchitecture and strength in the radius and tibia. Therefore, in postmenopausal women with a recent NVF, evaluation of morphometric VF can be used as a marker of generalized micro-architecture deterioration, independent of BMD. Future studies are needed to confirm our results and to determine the relevance of prevalent VF in addition to a NVF, in terms of subsequent VF and NVF risk.

Funding information This study was supported by funding of the Weijerhorst Foundation.

\section{Compliance with ethical standards}

The study protocol (registration number NL45707.072.13) was approved by an independent Medical Ethics Committee and complied with the Declaration of Helsinki. All patients gave written informed consent prior to participation.

Conflicts of interest Drs. Vranken has nothing to disclose.

Dr. Wyers has nothing to disclose.

Dr. van Rietbergen reports personal fees from Scanco Medical AG, outside the submitted work.

Dr. Driessen has nothing to disclose.
Dr. Geusens reports grants and other from Amgen, grants from Pfizer, grants from MSD, grants from UCB, grants from Abbott, grants and other from Lilly, grants from BMS, grants from Novartis, grants from Roche, and grants from Will Pharma, outside the submitted work.

Dr. Janzing has nothing to disclose.

Drs. Kaarsemaker has nothing to disclose.

Drs. van der Velde has nothing to disclose.

Dr. van den Bergh reports grants from Amgen, grants from Will Pharma, and grants from Eli Lilly, outside the submitted work.

Open Access This article is distributed under the terms of the Creative Commons Attribution-NonCommercial 4.0 International License (http:// creativecommons.org/licenses/by-nc/4.0/), which permits any noncommercial use, distribution, and reproduction in any medium, provided you give appropriate credit to the original author(s) and the source, provide a link to the Creative Commons license, and indicate if changes were made.

\section{References}

1. Melton LJ III, Lane AW, Cooper C, Eastell R, O'Fallon WM, Riggs BL (1993) Prevalence and incidence of vertebral deformities. Osteoporos Int 3:113-119. https://doi.org/10.1007/BF01623271

2. Cummings SR, Melton LJ (2002) Epidemiology and outcomes of osteoporotic fractures. Lancet 359:1761-1767. https://doi.org/10. 1016/S0140-6736(02)08657-9

3. Cauley JA, Palermo L, Vogt M, Ensrud KE, Ewing S, Hochberg M, Nevitt MC, Black DM (2008) Prevalent vertebral fractures in black women and white women. J Bone Miner Res 23:1458-1467. https://doi.org/10.1359/jbmr.080411

4. Cooper C, Atkinson EJ, MichaelO'Fallon W, Melton JL III (1992) Incidence of clinically diagnosed vertebral fractures: a populationbased study in Rochester, Minnesota, 1985-1989. J Bone Miner Res 7:221-227. https://doi.org/10.1002/jbmr.5650070214

5. Delmas PD, van de Langerijt L, Watts NB, Eastell R, Genant H, Grauer A, Cahall DL (2005) Underdiagnosis of vertebral fractures is a worldwide problem: the IMPACT study. J Bone Miner Res 20: 557-563. https://doi.org/10.1359/JBMR.041214

6. Fink HA, Milavetz DL, Palermo L, Nevitt MC, Cauley JA, Genant HK, Black DM, Ensrud KE, Fracture Intervention Trial Research Group (2005) What proportion of incident radiographic vertebral deformities is clinically diagnosed and vice versa? J Bone Miner Res 20:1216-1222. https://doi.org/10.1359/JBMR.050314

7. Werkgroep CBO, Richtlijn Osteoporose en Fractuurpreventie, derde herziening [Dutch]. (CBO, Utrecht, 2011)

8. Gallacher SJ, Gallagher AP, McQuillian C, Mitchell PJ, Dixon T (2006) The prevalence of vertebral fracture amongst patients presenting with non-vertebral fractures. Osteoporos Int 18:185-192. https://doi.org/10.1007/s00198-006-0211-1

9. Howat I, Carty D, Harrison J, Fraser M, McLellan AR (2007) Vertebral fracture assessment in patients presenting with incident nonvertebral fractures. Clin Endocrinol 67:923-930. https://doi. $\operatorname{org} / 10.1111 / j .1365-2265.2007 .02988 . x$

10. van der Velde RY, Bours SPG, Wyers CE, Lems WF, Geusens PPMM, van den Bergh JPW (2017) Effect of implementation of guidelines on assessment and diagnosis of vertebral fractures in patients older than 50 years with a recent non-vertebral fracture. Osteoporos Int 28:3017-3022. https://doi.org/10.1007/s00198017-4147-4

11. Black DM, Arden NK, Palermo L, Pearson J, Cummings SR (1999) Prevalent vertebral deformities predict hip fractures and new vertebral deformities but not wrist fractures. Study of Osteoporotic 
Fractures Research Group. J Bone Miner Res 14:821-828. https:// doi.org/10.1359/jbmr.1999.14.5.821

12. Delmas PD, Genant HK, Crans GG, Stock JL, Wong M, Siris E, Adachi JD (2003) Severity of prevalent vertebral fractures and the risk of subsequent vertebral and nonvertebral fractures: results from the MORE trial. Bone 33:522-532

13. Gallagher JC, Genant HK, Crans GG, Vargas SJ, Krege JH (2005) Teriparatide reduces the fracture risk associated with increasing number and severity of osteoporotic fractures. J Clin Endocrinol Metab 90:1583-1587. https://doi.org/10.1210/jc.2004-0826

14. Siris ES, Genant HK, Laster AJ, Chen P, Misurski DA, Krege JH (2007) Enhanced prediction of fracture risk combining vertebral fracture status and BMD. Osteoporos Int 18:761-770. https://doi. org/10.1007/s00198-006-0306-8

15. Krege JH, Wan X, Lentle BC, Berger C, Langsetmo L, Adachi JD, Prior JC, Tenenhouse A, Brown JP, Kreiger N, Olszynski WP, Josse RG, Goltzman D, Goltzman D, Kreiger N, Tenenhouse A, Godmaire S, Dumont S, Berger C, Zhou W, Joyce C, Kovacs C, Sheppard E, Kirkland S, Kaiser S, Stanfield B, Brown JP, Bessette L, Gendreau M, Anastassiades T, Towheed T, Matthews B, Josse B, Jamal S, Murray T, Gardner-Bray B, Adachi JD, Papaioannou A, Pickard L, Olszynski WP, Davison KS, Thingvold J, Hanley DA, Allan J, Prior JC, Patel M, Vigna Y, Andjelic N, Lentle B (2013) Fracture risk prediction: importance of age, $\mathrm{BMD}$ and spine fracture status. Bonekey Rep. 2:404. https://doi.org/10.1038/bonekey.2013. 138

16. Melton LJ III, Riggs BL, Keaveny TM et al (2010) Relation of vertebral deformities to bone density, structure, and strength. J Bone Miner Res 25:1922-1930. https://doi.org/10.1002/jbmr.150

17. Sornay-Rendu E, Cabrera-Bravo J-L, Boutroy S, Munoz F, Delmas PD (2009) Severity of vertebral fractures is associated with alterations of cortical architecture in postmenopausal women. J Bone Miner Res 24:737-743. https://doi.org/10.1359/jbmr.081223

18. Stein EM, Liu XS, Nickolas TL, Cohen A, McMahon DJ, Zhou B, Zhang C, Kamanda-Kosseh M, Cosman F, Nieves J, Guo XE, Shane E (2012) Microarchitectural abnormalities are more severe in postmenopausal women with vertebral compared to nonvertebral fractures. J Clin Endocrinol Metab 97:E1918-E1926. https://doi. org/10.1210/jc.2012-1968

19. Szulc P, Boutroy S, Vilayphiou N, Chaitou A, Delmas PD, Chapurlat R (2011) Cross-sectional analysis of the association between fragility fractures and bone microarchitecture in older men: the STRAMBO study. J Bone Miner Res 26:1358-1367. https:// doi.org/10.1002/jbmr.319

20. Sornay-Rendu E, Boutroy S, Duboeuf F, Chapurlat RD (2017) Bone microarchitecture assessed by HR-pQCT as predictor of fracture risk in postmenopausal women: the OFELY study. J Bone Miner Res 32:1243-1251. https://doi.org/10.1002/jbmr.3105

21. Biver E, Durosier-Izart C, Chevalley T, van Rietbergen B, Rizzoli R, Ferrari S (2018) Evaluation of radius microstructure and areal bone mineral density improves fracture prediction in postmenopausal women. J Bone Miner Res 33:328-337. https://doi.org/10. 1002/jbmr.3299

22. Langsetmo L, Peters KW, Burghardt AJ, Ensrud KE, Fink HA, Cawthon PM, Cauley JA, Schousboe JT, Barrett-Connor E, Orwoll ES, for the Osteoporotic Fractures in Men (MrOS) Study Research Group (2018) Volumetric bone mineral density and failure load of distal limbs predict incident clinical fracture independent of FRAX and clinical risk factors among older men. J Bone Miner Res 33:1302-1311. https://doi.org/10.1002/jbmr.3433

23. Szulc P, Boutroy S, Chapurlat R (2018) Prediction of fractures in men using bone microarchitectural parameters assessed by highresolution peripheral quantitative computed tomography-the prospective STRAMBO study. J Bone Miner Res 33:1470-1479. https://doi.org/10.1002/jbmr.3451
24. Samelson EJ, Broe KE, Xu H, Yang L, Boyd S, Biver E, Szulc P, Adachi J, Amin S, Atkinson E, Berger C, Burt L, Chapurlat R, Chevalley T, Ferrari S, Goltzman D, Hanley DA, Hannan MT, Khosla S, Liu CT, Lorentzon M, Mellstrom D, Merle B, Nethander M, Rizzoli R, Sornay-Rendu E, van Rietbergen B, Sundh D, Wong AKO, Ohlsson C, Demissie S, Kiel DP, Bouxsein ML (2019) Cortical and trabecular bone microarchitecture as an independent predictor of incident fracture risk in older women and men in the Bone Microarchitecture International Consortium (BoMIC): a prospective study. Lancet Diabetes Endocrinol 7:34-43. https://doi.org/10.1016/S22138587(18)30308-5

25. World Health Organization WHO FRAX WHO fracture risk assessment tool

26. Genant HK, Wu CY, van Kuijk C, Nevitt MC (1993) Vertebral fracture assessment using a semiquantitative technique. J Bone Miner Res 8:1137-1148. https://doi.org/10.1002/jbmr.5650080915

27. (2003) Prevention and management of osteoporosis. World Health Organ Tech Rep Ser 921:1-164- back cover

28. Pialat JB, Burghardt AJ, Sode M, Link TM, Majumdar S (2012) Visual grading of motion induced image degradation in high resolution peripheral computed tomography: impact of image quality on measures of bone density and micro-architecture. Bone 50:111118. https://doi.org/10.1016/j.bone.2011.10.003

29. Buie HR, Campbell GM, Klinck RJ, MacNeil JA, Boyd SK (2007) Automatic segmentation of cortical and trabecular compartments based on a dual threshold technique for in vivo micro-CT bone analysis. Bone 41:505-515. https://doi.org/10.1016/j.bone.2007. 07.007

30. Pistoia W, van Rietbergen B, Lochmüller E-M, Lill CA, Eckstein F, Rüegsegger P (2002) Estimation of distal radius failure load with micro-finite element analysis models based on three-dimensional peripheral quantitative computed tomography images. Bone 30 : 842-848

31. Dalzell N, Kaptoge S, Morris N, Berthier A, Koller B, Braak L, van Rietbergen B, Reeve J (2009) Bone micro-architecture and determinants of strength in the radius and tibia: age-related changes in a population-based study of normal adults measured with highresolution pQCT. Osteoporos Int 20:1683-1694. https://doi.org/ 10.1007/s00198-008-0833-6

32. Schousboe JT, Debold CR (2006) Reliability and accuracy of vertebral fracture assessment with densitometry compared to radiography in clinical practice. Osteoporos Int 17:281-289. https://doi.org/ 10.1007/s00198-005-2010-5

33. Lee J-H, Lee YK, Oh S-H, Ahn J, Lee YE, Pyo JH, Choi YY, Kim D, Bae SC, Sung YK, Kim DY (2016) A systematic review of diagnostic accuracy of vertebral fracture assessment (VFA) in postmenopausal women and elderly men. Osteoporos Int 27:16911699. https://doi.org/10.1007/s00198-015-3436-z

34. Schlenker RA, VonSeggen WW (1976) The distribution of cortical and trabecular bone mass along the lengths of the radius and ulna and the implications forin vivo bone mass measurements. Calcif Tissue Res 20:41-52. https://doi.org/10.1007/BF02546396

35. Boyd SK (2008) Site-specific variation of bone micro-architecture in the distal radius and tibia. J Clin Densitom 11:424 430. https:// doi.org/10.1016/j.jocd.2007.12.013

36. Shanbhogue VV, Hansen S, Halekoh U, Brixen K (2015) Use of relative vs fixed offset distance to define region of interest at the distal radius and tibia in high-resolution peripheral quantitative computed tomography. J Clin Densitom 18:217-225. https://doi. org/10.1016/j.jocd.2014.12.002

Publisher's note Springer Nature remains neutral with regard to jurisdictional claims in published maps and institutional affiliations. 